\title{
IDENTITIES INVOLVING ITERATED INTEGRAL TRANSFORMS
}

\author{
CYRIL NASIM \\ Department of Mathematics and Statistics \\ The University of Calgary \\ Calgary, Alberta Canada T2N $1 \mathrm{~N} 4$ \\ (Received August 18, 1982)
}

ABSTRACT. A number of identities involving iterated integral transforms are established, making use of the fact that a function which is a linear combination of the Macdonald's function $K_{v}(z)$, where $z$ is a complex variable, is a Fourier kernel.

KEY WORDS AND PHRASES. Macdonald's function, Fourier kernel, Mellin trans horm, Hankel transform, Laplace transform.

1982 MATHEMATICS SURJECT CLASSIFICATION CODE. 44 A20.

1. INTRODUCTION.

The object of this note is to establish various identities involving integral operators. The integral operators are the integral transforms with respect to the function $K_{v}(z)$, where $K_{\nu}(z)$ is the Macdonald's function of order $\nu$ and argument $z$, a complex variable. Some functional relations are deduced, as special cases, which show the inter-relations among more familiar Fourier Sine, Fourier Cosine, and Laplace transforms.

2. THE KERNEL.

Let $\quad y=x^{\frac{1}{2}} K{ }_{v}(\theta x)$, with $\theta$ a constant and $|\nu|<1$.

Then

$y^{\prime \prime}-\frac{v^{2}-1 / 4}{x^{2}} y=\theta^{2} y$

or

$$
\left(D^{2}-\frac{v^{2}-1 / 4}{x^{2}}\right) y=\theta^{2} y, \quad D \equiv \frac{d}{d x} .
$$

Whence

$$
\left(D^{2}-\frac{v^{2}-1 / 4}{x^{2}}\right)^{k} y=\theta^{2 k} y, \quad k=0,1,2, \ldots .
$$

Now, if we set $\theta=i e^{i \pi m / k}, 0 \leq m \leq 2 k-1$, 
then $\quad y=x^{\frac{1}{2}} K_{v}\left(i e^{i \pi m / k} x\right)$,

satisfies a $k$-fold Bessel equation:

$$
\left(D^{2}-\frac{v^{2}-1 / 4}{x^{2}}\right)^{k} y=(-1)^{k} y
$$

It is not difficult to see that if $x$ is a complex variable, then every point of (2.1) is regular except for a singularity at $x=0$. Now consider a function of the form

$$
G_{k}(x)=\sum_{m=0}^{2 k-1} B_{m} x^{\frac{1}{2} K_{v}}\left(i e^{i \pi m / k} x\right), \quad|v|<1 .
$$

The functions are an extension of the functions which were first noted by Guinand. As a special case when $k=2$, chose the coefficients as

$$
B_{0}=B_{2}=-\frac{1}{\pi}, \quad B_{1}=0 \text { and } B_{3}=-\frac{2}{\pi} \cos \frac{1}{2} \nu \pi \text {. }
$$

Then we obtain

$$
\begin{aligned}
G_{2}(x) & =-\frac{1}{\pi} x^{\frac{1}{2}}\left\{K_{\nu}(i x)+K_{\nu}(-i x)+2 \cos \left(\frac{1}{2} \nu \pi\right) K_{\nu}(x)\right\} \\
& =K_{\nu}(x), \text { say, }
\end{aligned}
$$

and we have

THEOREM 2.1. $y=k_{v}(x)$ is a solution of

$$
\left(D^{2}-\frac{v^{2}-1 / 4}{x^{2}}\right)^{2} y=y, \quad 0<x<\infty,
$$

the two-fold Bessel equation.

The function $k_{\nu}(x)$ is of special interest to us here and we shall develop its properties further.

Using the representations [3]

$$
K_{\nu}(x)=\frac{\pi}{2} \operatorname{cosec} v \pi\left\{I_{-\nu}(x)-I_{\nu}(x)\right\}
$$

and

$$
Y_{\nu}(x)=\operatorname{cosec} \nu \pi\left\{\cos \nu \pi J_{\nu}(x)-J_{-\nu}(x)\right\}
$$

where $J_{V}, I_{\nu}$ and $Y_{\nu}$ are the usual Bessel functions, equation (2.2), can be written as

$$
k_{\nu}(x)=x^{\frac{1}{2}}\left\{\sin \frac{1}{2} \nu \pi J_{\nu}(x)+\cos \frac{1}{2} \nu \pi\left(y_{\nu}(x)+\frac{2}{\pi} K_{\nu}(x)\right)\right\}
$$

These functions arise as kernels in divisor summation formulae of the Hardy-Landau 
type, involving number theoretic function $\sigma_{k}(n)$, the number of $k$ th powers of the divisor of $n,[4]$. If we put $\nu= \pm \frac{1}{2}$, we have

$$
k_{ \pm \frac{1}{2}}(x)=\pi^{-\frac{1}{2}}\left(\cos x-\sin x+e^{-x}\right) \text {, }
$$

which obviously satisfies the differential equation

$$
D^{4} y=y
$$

Next, the Mellin transform of $x^{\frac{1}{2}} K_{v}(\alpha x)$ is given by

$$
m\left\{x^{\frac{1}{2}} K_{\nu}(\alpha x)\right\}=\alpha^{-s-\frac{1}{2}} 2^{s-3 / 2} \Gamma\left(\frac{1}{2} s+\frac{1}{2} \nu+\frac{1}{4}\right) \Gamma\left(\frac{1}{2} s-\frac{1}{2} \nu+\frac{1}{4}\right) \text {, }
$$

where $\operatorname{Re} s>|\operatorname{Re} v|-\frac{1}{2},[5]$, whence the Mellin transform of $k_{v}(x)$ defined in (2.2) is given by

$$
\begin{aligned}
\underset{v}{k}(s)=-\frac{2^{s-3 / 2}}{\pi} \Gamma\left(\frac{1}{2} s+\right. & \left.\frac{1}{2} \nu+\frac{1}{4}\right) \Gamma\left(\frac{1}{2} s-\frac{1}{2} \nu+\frac{1}{4}\right) \\
& \left(i^{-s-\frac{1}{2}}+(-i)^{-s-\frac{1}{2}}+2 \cos \frac{1}{2} \nu \pi\right) .
\end{aligned}
$$

On simp1ifying, we have

$$
\begin{aligned}
\underset{v}{k *}(s)=-\frac{2^{s+\frac{1}{2}}}{\pi} \Gamma\left(\frac{1}{2} s+\frac{1}{2} v+\frac{1}{4}\right) \Gamma\left(\frac{1}{2} s-\frac{1}{2} \nu+\frac{1}{4}\right) \\
\quad \cos \frac{1}{4} \pi\left(s+\nu+\frac{1}{2}\right) \cdot \cos \frac{1}{4}\left(s-\nu+\frac{1}{2}\right),
\end{aligned}
$$

for

$$
\operatorname{Re} s>|\operatorname{Re} v|-\frac{1}{2} \text {. }
$$

By repeated use of the relation

$$
\Gamma(z) \Gamma(1-z)=\frac{\pi}{\sin \pi z},
$$

it is not a difficult matter to see that

$$
k_{v}^{*}(s) k_{v}^{*}(1-s)=1
$$

Hence,

THEOREM 2.2. The function $\underset{v}{k}(x)$ defined by equation (2.2) is a Fourier kernel, [6]. If we define the transformation $T[f]$ by 


$$
[f]=\int_{0}^{\infty} k_{\nu}(x t) f(t) d t, \quad|\nu|<1,
$$

then $r^{2}[f]=f$ and $T^{m}$ is involutory since $r^{2}=I$, the identity transformation. Making use of the asymptotic expansions of the Macdonald's function $k_{v}(z)$, we have

$\begin{aligned} k_{v}(x) & =0\left(e^{-x}\right), & x \rightarrow \infty \\ \text { and } & k_{v}(x)=0\left(x^{-v+\frac{1}{2}}\right), & x \rightarrow 0 .\end{aligned}$

Ther ef ore

$$
k_{v}(x) \in L^{2}(0, \infty), \quad \text { for }|v|<1
$$

Now, if we take $f(x) \in L^{2}(0, \infty)$, the integral defining the transformation $T$ exists and is in fact absolute convergent. Thus $T$ is a bounded transformation on $L^{2}$-space for $|v|<1$.

3. THE OPERATOR.

We shall now define the transformation $T$ in operator notation. Denote the operators $K_{v}$ and $K_{v, i}$ respectively by

$$
K_{v}[f] \equiv K_{v}\{f(x) ; x\}=\int_{0}^{\infty} \sqrt{x t} K_{v}(x t) f(t) d t
$$

and

$$
K_{v, i}[f] \equiv K_{v}\{f(x) ; i x\}=\int_{0}^{\infty} \sqrt{x t} K_{v}(i x t) f(t) d t,
$$

where $f \in L^{2}(0, \infty)$ and $|\nu|<1$, with $K_{\nu}(z)$ being the Macdonald's function. Then the transformation $T$ can be expressed, in operator form, as

$$
\begin{aligned}
T[f]= & \int_{0}^{\infty} k_{v}(x t) f(t) d t \\
= & -\frac{1}{\pi}\left\{\int_{0}^{\infty} \sqrt{x t} K(i x t) f(t) d t+\int_{0}^{\infty} \sqrt{x t} K(-i x t) f(t) d t\right. \\
& \left.+2 \cos \frac{1}{2} v \pi \int_{0}^{\infty} \sqrt{x t} K_{v}(x t) f(t) d t\right\} \\
= & -\frac{1}{\pi}\left(K_{v, i}+K_{v,-i}+2 \cos \frac{1}{2} v \pi K_{v}\right)[f] .
\end{aligned}
$$

and we can write, symbolically,

$$
T \equiv-\frac{1}{\pi}\left(K_{v, i}+K_{v,-i}+2 \cos \frac{1}{2} v \pi K_{v}\right)
$$


Since $T^{2}=I$, the identity transformation, we have

or

$$
\frac{1}{\pi^{2}}\left(K_{\nu, i}+K_{v,-i}+2 \cos \frac{1}{2} v \pi K_{v}\right)^{2}=I
$$

$$
\begin{aligned}
& I=\frac{1}{\pi^{2}}\left\{K_{v, i}^{2}+K_{v,-i}^{2}+4 \cos ^{2} \frac{1}{2} v \pi K_{v}^{2}+K_{v, i} K_{v,-i}+K_{v,-i} K_{v, i}\right. \\
& +2 \cos \frac{1}{2} \nu \pi\left(K_{\nu, i} K_{\nu}+K_{\nu,-i} K_{\nu}+K_{\nu} K_{\nu, i}+K_{\nu} K_{\nu,-i}\right\}
\end{aligned}
$$

The right-hand side is the linear combination of iterated transformations, which are bounded on $L^{2}$-space for $|\nu|<1$.

Now, using the standard result [5],

$$
\int_{0}^{\infty} t K_{v}(\alpha t) K_{v}(\beta t) d t=\frac{\pi(\alpha \beta)^{-v}}{\sin v \pi}\left(\frac{\alpha^{2 \nu}-\beta^{2} v}{\alpha^{2}-\beta^{2}}\right),
$$

where $|\nu|<1$ and $\operatorname{Re}(\alpha+\beta)>0$, we have for example, if $f \in L^{2}(0, \infty)$,

$$
\begin{aligned}
K_{v, i} K_{v}[f] & =\frac{1}{\pi^{2}} \int_{0}^{\infty} \sqrt{x t} K_{v}(i x t) d t \int_{0}^{\infty}(u t)^{\frac{1}{2}} K_{v}(u t) f(u) \\
& =\frac{1}{\pi^{2}} \int_{0}^{\infty}(x u)^{\frac{1}{2}} f(u) d u \int_{0}^{\infty} t K_{v}(i x t) K_{v}(u t) d t \\
& =\frac{-(i)^{-v}}{\pi \sin v \pi} \int_{0}^{\infty}(x u)^{\frac{1}{2}-v} f(u) \frac{(i x)^{2 v}-u^{2 v}}{x^{2}+u^{2}} d u \\
& =-K_{v} K_{v,-i}[f] .
\end{aligned}
$$

The change of order of integration can be justified by absolute convergence. Thus, we obtain our first identity

$$
K_{v, i} K_{v}+K_{v} K_{v,-i}=0
$$

The identity given by (3.2) can alternatively be established by making use of the Mellin transform theory. That is, the Mellin transform of the iterated operator $K_{v, i} K_{v}[f]$, is given forma1ly by

$$
\begin{aligned}
& m\left\{K_{\nu, i} K_{\nu}[f]=m\left\{-\frac{1}{\pi} x^{\frac{1}{2}} K_{v}(i x) ; s\right\} m\left\{-\frac{1}{\pi} x^{\frac{1}{2}} K_{v}(x) ; 1-s\right\} f *(s)\right. \\
& =\frac{i^{-s-\frac{1}{2}}}{4 \sin \frac{1}{2} \pi\left(s-v+\frac{1}{2}\right) \sin \frac{1}{2} \pi\left(s+v+\frac{1}{2}\right)} f *(s) \text {, }
\end{aligned}
$$

where $f *(s)$ denotes the Mellin transform of $f(x)$. Also,

$$
\begin{aligned}
m\left\{K_{\nu} K_{\nu,-i}\left[f_{j}\right\}\right. & =m\left\{-\frac{1}{\pi} x^{\frac{1}{2}} K_{\nu}(x) ; s\right\} m\left\{-\frac{1}{\pi} x^{\frac{1}{2}} K_{\nu}(-i x) ; 1-s\right\} f *(s) \\
& =\frac{(-i)^{s-3 / 2}}{4 \sin \frac{1}{2} \pi\left(s-\nu+\frac{1}{2}\right) \sin \frac{1}{2} \pi\left(s+\nu+\frac{1}{2}\right)}
\end{aligned}
$$


Then

$$
m\left\{\left(K_{v, i} K_{v}+K_{v} K_{v,-i}\right)[f]\right\}=0 \text {, }
$$

implying that

$$
K_{v, i} K_{v}+K_{v} K_{v,-i}=0
$$

as shown above. Similarly, one can show that

$$
K_{v} K_{v, i}+K_{v,-i} K_{v}=0
$$

a sort of conjugate of the identity in (3.2). Consider the representation

$$
K_{\nu}(i x)=\frac{\pi}{2 \sin \nu \pi}\left(e^{-i \frac{1}{2} \nu \pi} J_{-\nu}(x)-e^{i \frac{1}{2} \nu \pi_{e^{\top}}}(x)\right)
$$

then

$$
\begin{aligned}
& K_{v, i}[f]=\int_{0}^{\infty}(x t)^{\frac{1}{2}} K_{v}(i x t) f(t) d t, \quad|v|<1 \\
& =\frac{\pi}{2 \sin v \pi}\left\{e^{-i \frac{1}{2} \nu \pi} \int_{0}^{\infty}(x t)^{\frac{1}{2}} e_{-v}(x t) f^{\prime}(t) d t\right. \\
& \left.-e^{i \frac{1}{2} \nu \pi} \int_{0}^{\infty}(x t)^{\frac{1}{2}} J_{v}(x t) f(t) d t\right\} \\
& =\frac{\pi}{2 \sin \nu \tau_{i}}\left\{e^{-i \frac{1}{2} \nu \pi} H_{-\nu}[f]-e^{i \frac{1}{2} \nu \pi} H_{\nu}[f]\right\},
\end{aligned}
$$

where $H_{v}$ denotes the Hankel transform operator of order $v$. Thus, in operator form,

$$
K_{\nu, i}=\frac{\pi}{2 \sin \nu \pi} e^{-i \frac{1}{2} \nu \pi} H_{-\nu}-\frac{\pi}{2 \sin \nu \pi} e^{i \frac{1}{2} \nu \pi} H_{\nu}
$$

and similarly

$$
K_{\nu,-i}=\frac{\pi}{2 \sin \nu \pi} e^{i \frac{1}{2} \nu \pi} H_{-\nu}-\frac{\pi}{2 \sin v \pi} e^{-i \frac{1}{2} \nu \pi} H_{\nu} .
$$

Now, after substituting for $K_{v, i}$ and $K_{v,-i}$ in (3.2) and rearranging, we have

$$
e^{-i \frac{1}{2} \nu \pi}\left(H_{-v} K_{v}-K_{\nu} H_{v}\right)=e^{i \frac{1}{2} \nu \pi}\left(H_{v} K_{v}-K_{\nu} H_{-v}\right) \text {. }
$$

By comparing the real and imaginary parts and solving, we obtain the identities

$$
K_{v} H_{v}=H_{-v} K_{v}
$$

and

$$
H_{v} K_{\nu}=K_{\nu} H_{-v}
$$


Now,

$$
\begin{aligned}
H_{\frac{1}{2}}[f] & =\int_{0}^{\infty}(x t)^{\frac{1}{2}} J_{\frac{1}{2}}(x t) f(t) d t \\
& =\left(\frac{2}{\pi}\right)^{\frac{1}{2}} \int_{0}^{\infty} \sin (x t) f(t) d t \\
& =S[f],
\end{aligned}
$$

and similarly,

$$
H_{-\frac{1}{2}}[f]=C[f],
$$

where $S$ and $C$ are the usual Fourier since and cosine transform repsectively. Also,

$$
\begin{aligned}
K_{\frac{1}{2}}[f] & =\int_{0}^{\infty}(x t)^{\frac{1}{2}} K_{\frac{1}{2}}(x t) f(t) d t \\
& =\left(\frac{\pi}{2}\right)^{\frac{1}{2}} \int_{0}^{\infty} e^{-x t} f(t) d t \\
& =\left(\frac{\pi}{2}\right)^{\frac{1}{2}} L[f],
\end{aligned}
$$

where $L[f]$ is the Laplace transform.

Hence, setting $v= \pm \frac{1}{2}$ in $(3.4)$, we obtain the relations,

and

$$
E S=C L
$$

$$
L C=S L \text {. }
$$

Incidently, a general relation involving the operators $K_{\nu}$ and $H_{\mu}$ can be established:

$$
H_{\mu} K_{\nu}=\operatorname{cosec} \nu \pi\left\{\sin \frac{1}{2}(\nu-\mu) \pi K_{\mu} H_{\nu}+\sin \frac{1}{2}(\nu+\mu) \pi K_{\mu} H_{-\nu}\right.
$$

On setting $\mu= \pm \nu,(3.8)$ yields the identities. (3.5) and (3.6). Next, from the representation (3.4), we have symbolically

$$
\begin{aligned}
k_{\nu, i}^{2} & =\left(\frac{\pi}{2 \sin v \pi}\right)^{2}\left(e^{-i \frac{1}{2} \nu \pi} H_{-\nu}-e^{i \frac{1}{2} \nu \pi} H_{\nu}\right)^{2} \\
& =\left(\frac{\pi}{2 \sin v \pi}\right)^{2}\left(2 \cos v \pi I-H_{-\nu} H_{\nu}-H_{\nu} H_{-\nu}\right)
\end{aligned}
$$

since $H_{-V}^{2}=H_{V}^{2}=I$, the identity operator, with $H_{v}$ being the Hankel transform. Similarly, one can show that

$$
R_{v, i}^{2}=K_{v,-i}^{2}
$$


And, in the same vein, we have

$$
K_{\nu, i} K_{\nu,-i}+K_{\nu,-i} K_{\nu, i}=2\left(\frac{\pi}{2 \sin v \pi}\right)^{2}\left\{2 I-\cos v \pi\left(H_{\nu} H_{-\nu}+H_{-\nu} H_{\nu}\right)\right\}
$$

Now, going back to equation (3.1) and using the results given by (3.2), (3.3), and (3.9) in (3.11) and simplifying, we have finally, for $|\nu|<1$,

$$
H_{\nu} H_{-\nu}+H_{-\nu} H_{\nu}-\left(\frac{2}{\pi} \sin v \pi\right)^{2} K_{\nu}^{2}=2 \cos v \pi I \text {. }
$$

An interesting relationship can be established by putting $v= \pm \frac{1}{2}$ in (3.12). Then

$$
H_{\frac{1}{2}} H_{-\frac{1}{2}}+H_{-\frac{1}{2}} H_{1 / 2}-\frac{4}{\pi^{2}} \quad K_{\frac{1}{2}}^{2}=0
$$

or

$$
S C+C S=\frac{2}{\pi} L^{2}
$$

where $S, C$, and $L$ denote the Fourier sine, Fourier consine and Laplace transforms respectively.

From (3.9) and (3.12), one can establish the identity

$$
K_{v}^{2}=-K_{v, i}^{2}=-K_{v,-i}^{2}
$$

which, on setting $v= \pm \frac{1}{2}$, yields (3.13).

\section{REFERENCRS}

1. GIINAND, A.P., A class of Fourier Kernels, Quart. J. Math. Oxford (2), 1 (1950), 191-3.

2. EVERITT, W.N. On a generalization of Bessel functions and resulting class of Fourier kernels, Quart. J. Math. Oxford (2), 10 (1959), 270-9.

3. WATSON, G.N., Theory of Bessel functions. The University Press, Cambridge, England, Second Edition, 1944.

4. NASIM, C. A summation formula involving $\sigma_{k}(n), k>1$, Can. J. Math. 21 (1960) 951-64.

5. ERDELYI et a1., A., Tables of Integral Transforms, Vol. I, Bateman Manuscript Project, McGraw-Hil1 Book Co., New York, 1954.

6. TITCHMARSH, E.C., Introduction to the theory of rourier Integrals, The Claredon Press, Oxford, Second Edition, 1948. 


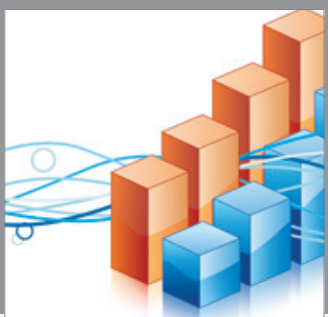

Advances in

Operations Research

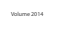

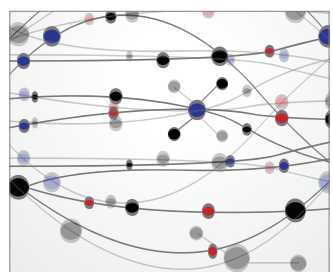

\section{The Scientific} World Journal
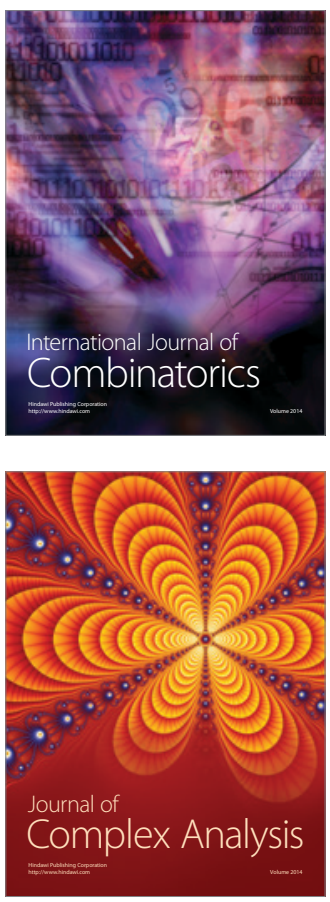

International Journal of

Mathematics and

Mathematical

Sciences
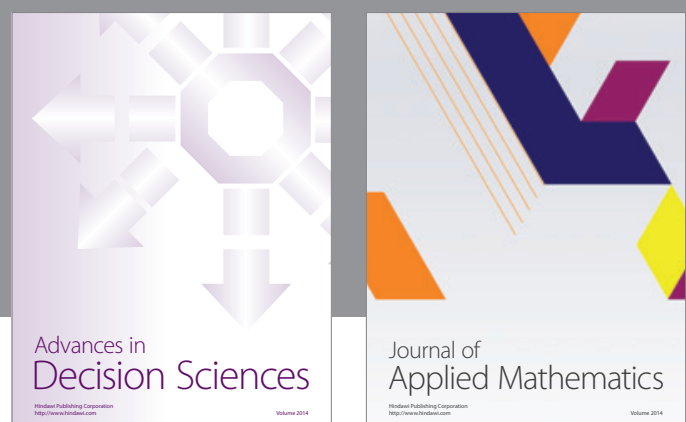

Journal of

Applied Mathematics
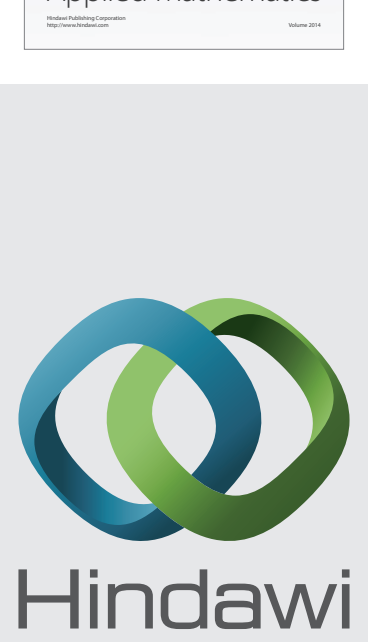

Submit your manuscripts at http://www.hindawi.com
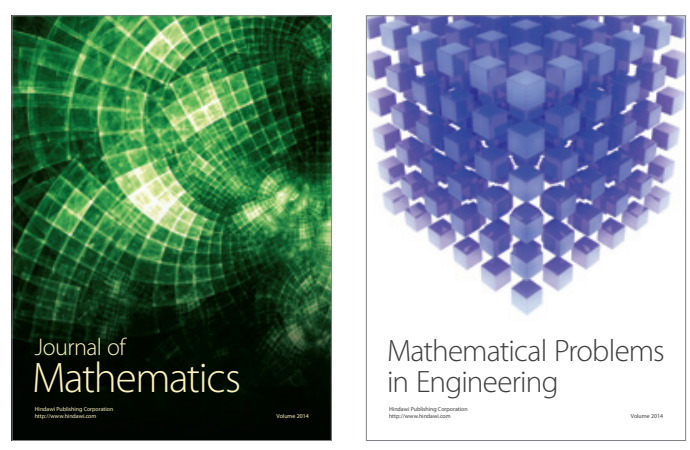

Mathematical Problems in Engineering
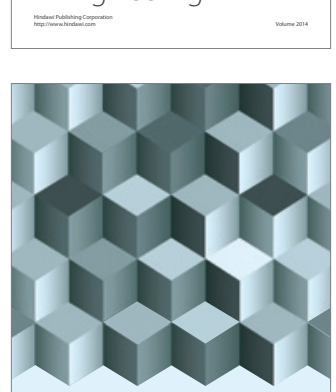

Journal of

Function Spaces
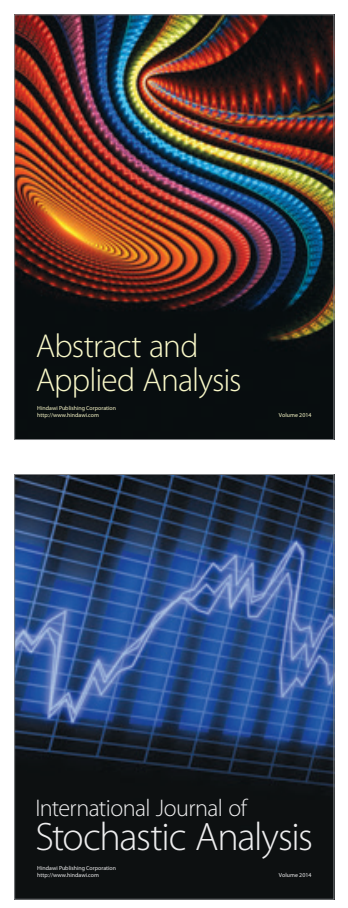

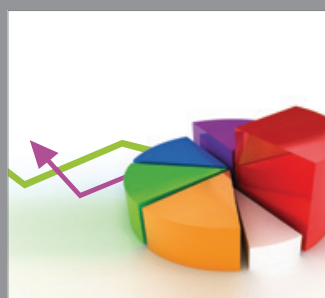

ournal of

Probability and Statistics

Promensencen
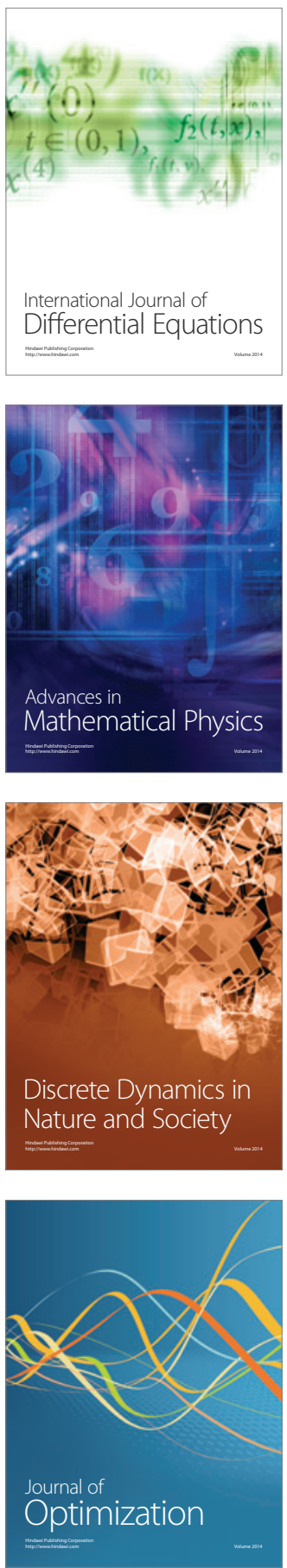